\title{
Many Touchings Force Many Crossings
}

\author{
János Pach ${ }^{1,2}$ and Géza Tóth ${ }^{2}$ \\ 1 École Polytechnique Fédérale de Lausanne, St. 8, Lausanne 1015, Switzerland \\ pach@cims.nyu.edu \\ 2 Rényi Institute, Hungarian Academy of Sciences 1364 Budapest, POB 127, \\ Hungary \\ geza@renyi.hu
}

\begin{abstract}
Given $n$ continuous open curves in the plane, we say that a pair is touching if they have only one interior point in common and at this point the first curve does not get from one side of the second curve to its other side. Otherwise, if the two curves intersect, they are said to form a crossing pair. Let $t$ and $c$ denote the number of touching pairs and crossing pairs, respectively. We prove that $c \geq \frac{1}{10^{5}} \frac{t^{2}}{n^{2}}$, provided that $t \geq 10 n$. Apart from the values of the constants, this result is best possible.
\end{abstract}

Keywords: planar curves, touching, crossing

\section{Introduction}

In the context of the theory of topological graphs and graph drawing, many interesting questions have been raised concerning the adjacency structure of a family of curves in the plane or in another surface [5]. In particular, during the past four decades, various important properties of string graphs (i.e., intersection graphs of curves in the plane) have been discovered, and the study of different crossing numbers of graphs and their relations to one another has become a vast area of research. A useful tool in these investigations is the so-called crossing lemma of Ajtai, Chvátal, Newborn, Szemerédi and Leighton [1, 7]. It states the following: Given a graph of $n$ vertices and $e>4 n$ edges, no matter how we draw it in the plane by not necessarily straight-line edges, there are at least constant times $e^{3} / n^{2}$ crossing pairs of edges.

This lemma has inspired a number of results establishing the existence of many crossing subconfigurations of a given type in sufficiently rich geometric or topological structures [2], 10, [1], 6].

In this note, we will be concerned with families of curves in the plane. By a curve, we mean a non-selfintersecting continuous arc in the plane, that is, a homeomorphic image of the open interval $(0,1)$. Two curves are said to touch each other if they have precisely one interior point in common and at this point the first curve does not pass from one side of the second curve to the other. Any other pair of curves with nonempty intersection is called crossing. A family of 
curves is in general position if any two of them intersect in a finite number of points and no three pass through the same point.

Let $n$ be even, $t$ be a multiple of $n$, and suppose that $n \leq t<\frac{n^{2}}{4}$. Consider a collection $A$ of $n-\frac{2 t}{n}>\frac{n}{2}$ pairwise disjoint curves, and another collection $B$ of $\frac{2 t}{n}$ curves such that

(i) $A \cup B$ is in general position,

(ii) each element of $B$ touches precisely $\frac{n}{2}$ elements of $A$, and

(iii) no two elements of $B$ touch each other.

The family $A \cup B$ consists of $n$ curves such that the number of touching pairs among them is $t$. The only pairs of curves that may cross each other belong to $B$. Thus, the number of crossing pairs is at most $\left(\begin{array}{c}2 t / n \\ 2\end{array}\right) \leq \frac{2 t^{2}}{n^{2}}$. See Figure 1 .

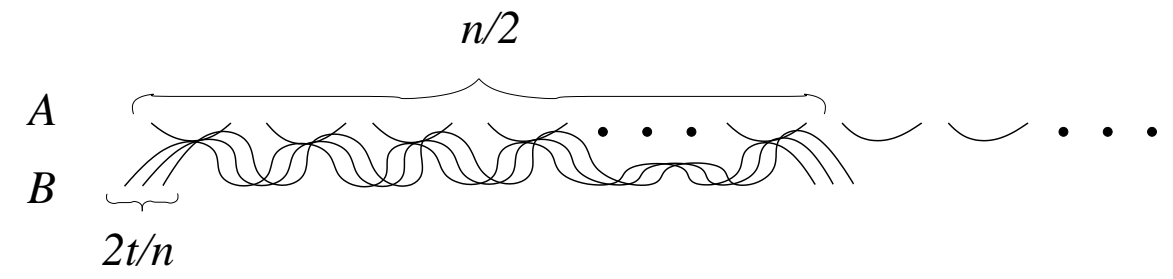

Fig. 1. A set of $n$ curves with $t$ touching pairs and at most $\frac{2 t^{2}}{n^{2}}$ crossing pairs.

The aim of the present note is to prove that this construction is optimal up to a constant factor, that is, any family of $n$ curves and $t$ touchings has at least constant times $\frac{t^{2}}{n^{2}}$ crossing pairs.

Theorem. Consider a family of $n$ curves in general position in the plane which determines $t$ touching pairs and c crossing pairs.

If $t \geq 10 n$, then we have $c \geq \frac{1}{10^{5}} \frac{t^{2}}{n^{2}}$. This bound is best possible up to a constant factor.

We make no attempt to optimize the constants in the theorem.

Pach, Rubin, and Tardos 8 established a similar relationship between $t$, the number of touching pairs, and $C$, the number of crossing points between the curves. They proved that $C \geq t(\log \log (t / n))^{\delta}$, for an absolute constant $\delta>0$. Obviously, we have $C \geq c$. There is an arrangement of $n$ red curves and $n$ blue curves in the plane such that every red curve touches every blue curve, and the total number of crossing points is $C=\Theta\left(n^{2} \log n\right)$; cf. 4]. Of course, the number of crossing pairs, $c$, can never exceed $\left(\begin{array}{l}n \\ 2\end{array}\right)$.

Between $n$ arbitrary curves, the number of touchings $t$ can be as large as $\left(\frac{3}{4}+o(1)\right)\left(\begin{array}{l}n \\ 2\end{array}\right)$; cf. [9]. However, if we restrict our attention to algebraic plane curves of bounded degree, then we have $t=O\left(n^{3 / 2}\right)$, where the constant hidden in the notation depends on the degree [3]. 


\section{Proof of Theorem}

We start with an easy observation.

Lemma. Given a family of $n \geq 3$ curves in general position in the plane, no two of which cross, the number of touchings, $t$, cannot exceed $3 n-6$.

Proof. Pick a different point on each curve. Whenever two curves touch each other at a point $p$, connect them by an edge (arc) passing through $p$. In the resulting drawing, any two edges that do not share an endpoint are represented by disjoint arcs. According to the Hanani-Tutte theorem [13, this means that the underlying graph is planar, so that its number of edges, $t$, satisfies $t \leq 3 n-6$.

Proof of Theorem. We proceed by induction on $n$. For $n \leq 20$, the statement is void. Suppose that $n>20$ and that the statement has already been proved for all values smaller than $n$.

We distinguish two cases.

CASE A: $t \leq 10 n^{3 / 2}$.

In this case, we want to establish the stronger statement

$$
c \geq \frac{1}{10^{4}} \frac{t^{2}}{n^{2}} .
$$

By the assumption, we have

$$
\frac{1}{10^{4}} \frac{t^{2}}{n^{2}} \leq \frac{n}{100} .
$$

Let $G_{t}$ (resp., $G_{c}$ ) denote the touching graph (resp., crossing graph) associated with the curves. That is, the vertices of both graphs correspond to the curves, and two vertices are connected by an edge if and only if the corresponding curves are touching (resp., crossing).

Let $T$ be a minimal vertex cover in $G_{c}$, that is, a smallest set of vertices of $G_{c}$ such that every edge of $G_{c}$ has at least one endpoint in $T$. Let $\tau=|T|$. Let $U$ denote the complement of $T$. Obviously, $U$ is an independent set in $G_{c}$. According to the Lemma, the number of edges in $G_{t}[U]$, the touching graph induced by $U$, satisfies

$$
\left|E\left(G_{t}[U]\right)\right|<3|U| \leq 3 n .
$$

By the minimality of $T, G_{c}$ has at least $|T|=\tau$ edges. That is, we have $c \geq \tau$, so we are done if $\tau \geq \frac{1}{10^{4}} \frac{t^{2}}{n^{2}}$.

From now on, we can and shall assume that $\tau<\frac{1}{10^{4}} \frac{t^{2}}{n^{2}}$. By (1), we have $\frac{1}{10^{4}} \frac{t^{2}}{n^{2}} \leq \frac{n}{100}$. Hence, $|T| \leq \frac{n}{100}$ and

$$
|U|=n-|T| \geq \frac{99 n}{100} .
$$




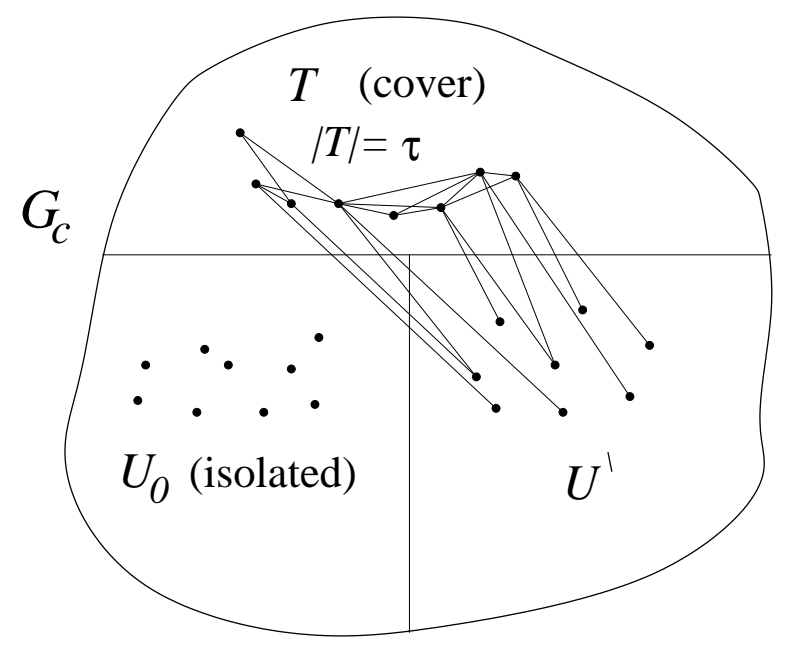

Fig. 2. Graph $G_{c}$.

Let $U^{\prime} \subseteq U$ denote the set of all vertices in $U$ that are not isolated in the graph $G_{c}$. By the definition of $T$, all neighbors of a vertex $v \in U$ in $G_{c}$ belong to $T$. If $\left|U^{\prime}\right| \geq \frac{1}{10^{4}} \frac{t^{2}}{n^{2}}$, then we are done, because $c \geq\left|U^{\prime}\right|$.

Therefore, we can assume that

$$
\left|U^{\prime}\right|<\frac{1}{10^{4}} \frac{t^{2}}{n^{2}} \leq \frac{n}{100},
$$

where the second inequality follows again by (1).

Letting $U_{0}=U \backslash U^{\prime}$, by (3) and (4) we obtain $\left|U_{0}\right|=|U|-\left|U^{\prime}\right| \geq \frac{98 n}{100}$. Clearly, all vertices in $U_{0}$ are isolated in $G_{c}$.

Suppose that $G_{t}\left[T \cup U^{\prime}\right]$ has at least $\frac{t}{10}$ edges. Consider the set of curves $T \cup U^{\prime}$. We have $n_{0}=\left|T \cup U^{\prime}\right| \leq \frac{2 n}{100}$ and, the number of touchings, $t_{0}=$ $\left|E\left(G_{t}\left[T \cup U^{\prime}\right]\right)\right| \geq \frac{t}{10}$. Therefore, by the induction hypothesis, for the number of crossings we have $c_{0}=\left|E\left(G_{c}\left[T \cup U^{\prime}\right]\right)\right| \geq \frac{1}{10^{5}} \frac{t_{0}^{2}}{n_{0}^{2}} \geq \frac{1}{10^{4}} \frac{t^{2}}{n^{2}}$ and we are done. Hence, we assume in the sequel that $G_{t}\left[T \cup U^{\prime}\right]$ has fewer than $\frac{t}{10}$ edges.

Consequently, for the number of edges in $G_{t}$ running between $T$ and $U_{0}$, we have

$$
\left|E\left(G_{t}\left[T, U_{0}\right]\right)\right| \geq t-\left|E\left(G_{t}\left[T \cup U^{\prime}\right]\right)\right|-\left|E\left(G_{t}\left[U_{0} \cup U^{\prime}\right]\right)\right| \geq t-\frac{t}{10}-3 n>\frac{t}{2} .
$$

Here we used the assumption that $t \geq 10 n$.

Let $\chi=\chi\left(G_{c}[T]\right)$ denote the chromatic number of $G_{c}[T]$. In any coloring of a graph with the smallest possible number of colors, there is at least one edge between any two color classes. Hence, $G_{c}[T]$ has at least $\left(\begin{array}{l}\chi \\ 2\end{array}\right) \geq \frac{1}{10^{4}} \frac{t^{2}}{n^{2}}$ edges, and we are done, provided that $\chi>\frac{1}{70} \cdot \frac{t}{n}$. 
Thus, we can suppose that

$$
\chi=\chi\left(G_{c}[T]\right) \leq \frac{1}{70} \cdot \frac{t}{n} .
$$

Consider a coloring of $G_{c}[T]$ with $\chi$ colors, and denote the color classes by $I_{1}, I_{2}, \ldots, I_{\chi}$. Obviously, for every $j, I_{j} \cup U_{0}$ is an independent set in $G_{c}$. Therefore, by the Lemma, $G_{t}\left[I_{j} \cup U_{0}\right]$ has at most $3 n$ edges. Summing up for all $j$ and taking (6) into account, we obtain

$$
\left|E\left(G_{t}\left[T, U_{0}\right]\right)\right| \leq \sum_{j=1}^{\chi}\left|E\left(G_{t}\left[I_{j} \cup U_{0}\right]\right)\right| \leq \frac{1}{70} \cdot \frac{t}{n} 3 n \leq \frac{t}{20},
$$

contradicting (5). This completes the proof in CASE A.

CASE B: $t \geq 10 n^{3 / 2}$. Set $p=\frac{10 n^{3}}{t^{2}} \leq \frac{1}{10}$. Select each curve independently with probability $p$. Let $\mathbf{n}^{\prime}, \mathbf{t}^{\prime}$, and $\mathbf{c}^{\prime}$ denote the number of selected curves, the number of touching pairs, and the number of crossing pairs between them, respectively. Clearly,

$$
E\left[\mathbf{n}^{\prime}\right]=p n, \quad E\left[\mathbf{t}^{\prime}\right]=p^{2} t, \quad E\left[\mathbf{c}^{\prime}\right]=p^{2} c .
$$

The number of selected curves, $\mathbf{n}^{\prime}$, has binomial distribution, therefore,

$$
\operatorname{Prob}\left[\left|\mathbf{n}^{\prime}-p n\right|>\frac{1}{4} p n\right]<\frac{1}{3} .
$$

By Markov's inequality,

$$
\operatorname{Prob}\left[\mathbf{c}^{\prime}>3 p^{2} c\right]<\frac{1}{3}
$$

Consider the touching graph $G_{t}$. Let $d_{1}, \ldots, d_{n}$ denote the degrees of the vertices of $G_{t}$, and let $e_{1}, \ldots, e_{t}$ denote its edges, listed in any order. We say that an edge $e_{i}$ is selected (or belongs to the random sample) if both of its endpoints were selected. Let $X_{i}$ be the indicator variable for $e_{i}$, that is,

$$
X_{i}= \begin{cases}1 & \text { if } e_{i} \text { was selected } \\ 0 & \text { otherwise }\end{cases}
$$

We have $E\left[X_{i}\right]=p^{2}$. Let $\mathbf{t}^{\prime}=\sum_{i=1}^{t} X_{i}$. It follows by straightforward computation that for every $i$,

$$
\operatorname{var}\left[X_{i}\right]=E\left[\left(X_{i}-E\left[X_{i}\right]\right)^{2}\right]=p^{2}-p^{4},
$$

If $e_{i}$ and $e_{j}$ have a common endpoint for some $i \neq j$, then

$$
\operatorname{cov}\left[X_{i}, X_{j}\right]=E\left[X_{i} X_{j}\right]-E\left[X_{i}\right] E\left[X_{j}\right]=p^{3}-p^{4} .
$$

If $e_{i}$ and $e_{j}$ do not have a common vertex, then $X_{i}$ and $X_{j}$ are independent random variables and $\operatorname{cov}\left[X_{i}, X_{j}\right]=0$. Therefore, we obtain

$$
\sigma^{2}=\operatorname{var}\left[\mathbf{t}^{\prime}\right]=\sum_{i=1}^{t} \operatorname{var}\left[X_{i}\right]+\sum_{1 \leq i \neq j \leq t} \operatorname{cov}\left[X_{i}, X_{j}\right]
$$




$$
=\left(p^{2}-p^{4}\right) t+\left(p^{3}-p^{4}\right) \sum_{i=1}^{n} d_{i}\left(d_{i}-1\right)<p^{2} t+2 p^{3} n t .
$$

From here, we get $\sigma<\sqrt{p^{2} t}+\sqrt{2 p^{3} n t}<p^{2} t=E\left[\mathbf{t}^{\prime}\right]$. By Chebyshev's inequality,

$$
\operatorname{Prob}\left[\left|\mathbf{t}^{\prime}-p^{2} t\right| \geq \lambda \sigma\right] \leq \frac{1}{\lambda^{2}}
$$

Setting $\lambda=\frac{1}{4}$,

$$
\operatorname{Prob}\left[\left|\mathbf{t}^{\prime}-p^{2} t\right| \geq \frac{p^{2} t}{4}\right] \leq \frac{1}{4^{2}}<\frac{1}{3} .
$$

It follows from (8), (9), and (10) that, with positive probability, we have

$$
\left|\mathbf{n}^{\prime}-p n\right| \leq \frac{1}{4} p n, \quad \mathbf{c}^{\prime} \leq 3 p^{2} c, \quad\left|\mathbf{t}^{\prime}-p^{2} t\right| \leq \frac{1}{4} p^{2} t .
$$

Consider a fixed selection of $n^{\prime}$ curves with $t^{\prime}$ touching pairs and $c^{\prime}$ crossing pairs for which the above three inequalities are satisfied. Then we have

$$
\begin{aligned}
& t^{\prime} \geq \frac{3}{4} p^{2} t=\frac{300}{4} \cdot \frac{n^{6}}{t^{3}}, \\
& n^{\prime} \leq \frac{5}{4} p n=\frac{50}{4} \cdot \frac{n^{4}}{t^{2}}
\end{aligned}
$$

and, hence,

$$
t^{\prime} \geq \frac{6 n^{2}}{t} n^{\prime} \geq 10 n^{\prime}
$$

On the other hand,

$$
\begin{aligned}
& t^{\prime} \leq \frac{5}{4} p^{2} t=\frac{500}{4} \cdot \frac{n^{6}}{t^{3}}, \\
& n^{\prime} \geq \frac{3}{4} p n=\frac{30}{4} \cdot \frac{n^{4}}{t^{2}}
\end{aligned}
$$

so that

$$
10\left(n^{\prime}\right)^{3 / 2} \geq 10 \cdot \frac{30^{3 / 2}}{4^{3 / 2}} \cdot \frac{n^{6}}{t^{3}}>t^{\prime} .
$$

According to (12) and (13), the selected family meets the requirements of the Theorem in CASE A. Thus, we can apply the Theorem in this case to obtain that $c^{\prime} \geq \frac{1}{10^{4}} \frac{t^{\prime 2}}{n^{\prime 2}}$. In view of (11), we have

$$
3 p^{2} c \geq c^{\prime}, \quad t^{\prime} \geq \frac{3}{4} p^{2} t, \quad n^{\prime} \leq \frac{5}{4} p n .
$$

Thus,

$$
3 p^{2} c \geq c^{\prime} \geq \frac{1}{10^{4}} \frac{t^{\prime 2}}{n^{\prime 2}} \geq \frac{1}{10^{4}} \frac{\left(3 p^{2} t / 4\right)^{2}}{(5 p n / 4)^{2}}=\frac{1}{10^{4}}\left(\frac{3}{5}\right)^{2} \frac{p^{2} t^{2}}{n^{2}} .
$$


Comparing the left-hand side and the right-hand side, we conclude that

$$
c \geq \frac{1}{10^{5}} \frac{t^{2}}{n^{2}}
$$

as required. This completes the proof of the Theorem.

Acknowledgment. The work of János Pach was partially supported by Swiss National Science Foundation Grants 200021-165977 and 200020-162884. Géza Tóth's work was partially supported by the Hungarian National Research, Development and Innovation Office, NKFIH, Grant K-111827.

\section{References}

1. Ajtai, M., Chvátal, V., Newborn, M., Szemerédi, E.: Crossing-free subgraphs. In: Theory and Practice of Combinatorics, North-Holland Mathematics Studies 60, North-Holland, Amsterdam, 1982, 9-12.

2. Dey, T. K.: Improved bounds on planar $k$-sets and related problems, Discrete Comput. Geom. 19 (1998), 373-382.

3. Ellenberg, J. S., Solymosi, J., Zahl, J.: New bounds on curve tangencies and orthogonalities, Discrete Anal. (2016), Paper No. 18, 22 pp.

4. Fox, J., Frati, F., Pach, J., Pinchasi, R.: Crossings between curves with many tangencies, in: WALCOM: Algorithms and Computation, Lecture Notes in Comput. Sci. 5942, Springer-Verlag, Berlin, 2010, 1-8. Also in: An Irregular Mind, Bolyai Soc. Math. Stud. 21, János Bolyai Math. Soc., Budapest, 2010, 251-260.

5. Fox, J., Pach, J.: A separator theorem for string graphs and its applications, Combin. Probab. Comput. 19 (2010), 371-390.

6. García, A., Noy, M., Tejel, J.: Lower bounds on the number of crossing-free subgraphs of $K_{n}$, Comput. Geom. 16 (2000), no. 4, 211-221.

7. Leighton, T.: Complexity Issues in VLSI, Foundations of Computing Series, MIT Press, Cambridge, 1983.

8. Pach, J., Rubin, N., Tardos, G.: Beyond the Richter-Thomassen Conjecture, in: Proc. 27th Annual ACM-SIAM Symposium on Discrete Algorithms (SODA 2016, Arlington), SIAM, 2016, 957-968.

9. Pach, J., Tóth, G.: How many ways can one draw a graph? Combinatorica 26 (2006), no. 5, 559-576.

10. Sharir, M.: The Clarkson-Shor technique revisited and extended, Combin. Probab. Comput. 12 (2003), no. 2, 191-201.

11. Solymosi, J., Tóth, C. D.: Distinct distances in the plane, Discrete Comput. Geom. 25 (2001), no. 4, 629-634.

12. Székely, L. A.: Crossing numbers and hard Erdős problems in discrete geometry, Combin. Probab. Comput. 6 (1997), no. 3, 353-358.

13. Tutte, W. T.: Toward a theory of crossing numbers, J. Combinatorial Theory 8 (1970), 45-53. 